\title{
Magnetite layer formation in the Bushveld Complex of South Africa
}

\section{Zhuo-Sen Yao}

Carleton University

James Mungall ( $\nabla$ jamesmungall@cunet.carleton.ca )

Carleton University https://orcid.org/0000-0001-9726-8545

\section{Article}

Keywords: magnetite layer formation, South Africa, Bushveld Complex

Posted Date: July 13th, 2021

DOl: https://doi.org/10.21203/rs.3.rs-673073/v1

License: (c) (i) This work is licensed under a Creative Commons Attribution 4.0 International License. Read Full License

Version of Record: A version of this preprint was published at Nature Communications on January 20th, 2022. See the published version at https://doi.org/10.1038/s41467-022-28000-9. 


\section{Abstract}

6 The great economic significance of layered mafic-ultramafic intrusions like the Bushveld

7 Complex of South Africa results from the existence within them of some layers highly

8 concentrated in valuable elements. Here we address the origins of the Main Magnetite Layer,

9 a globally important resource of Fe-Ti-V-rich magnetite. Previous models of in situ

10 fractional magnetite crystallization require frequent ad hoc adjustments to the boundary

\section{Magnetite layer formation in the Bushveld Complex of South Africa}

\author{
Zhuo-sen Yao and James E. Mungall*
}

Department of Earth Sciences, Carleton University, Ottawa, Ontario K1S 5B6, Canada conditions. An alternative model of rapid deposition of loose piles of magnetite crystals followed by compositional convection near the top of the pile and infiltration of the pile from beneath by migrating intercumulus melt fits observations without any adjustments. The data admit both explanations, but the latter model, with the fewest unconstrained interventions, is preferable. The choice of models has pivotal ramifications for understanding of the fundamental processes by which crystals accumulate and layers form in layered intrusions.

\section{Introduction}

Because it is impossible to observe directly the processes that produce layered plutonic mafic rocks, the nature of these processes must be inferred from the record of composition, mineral mode, and texture of the final products of emplacement, crystallization, and cooling of the magma ${ }^{1}$. Even the most basic of questions, such as whether or not crystals form within the melt and settle to the floor ${ }^{2-3}$ or are nucleated and grow in situ (i.e., by heterogeneous nucleation in direct physical contact with the solid base $)^{4-5}$, remain open to controversy after a century of dedicated research effort. Progress will be difficult without resolution of such 
fundamental questions. A common approach to attempt to resolve these matters is to produce

27 a forward geochemical or physical model and use it to demonstrate the feasibility of a particular hypothetical process; however, there are many examples of cases where totally contradictory hypotheses can be framed in forward models that successfully match observations $^{6-7}$. The resolution of these questions is of more than purely academic interest because some of the layers in question contain mineral deposits of global economic significance $^{8}$. It is therefore essential to address the larger framework within which individual hypotheses are framed before we can make reasoned judgements about which ones to favor.

One deposit that has long been used as a laboratory to address the controls on layer formation in layered intrusions is the Main Magnetite Layer (MML) in the Upper Zone of the Rustenburg Layered Suite of the Bushveld Complex of South Africa ${ }^{9-19}$. Here we compare observations of the MML with some forward thermodynamic and fluid dynamic models, appealing to various combinations of fractional crystallization, compositional convection during adcumulus crystal growth, and reactive transport of upward-migrating Cr-rich melt from beneath the MML. By comparison of our models with previously published work we show that multiple model approaches can achieve similar results, with the result that no model can be used definitively to exclude any other; however, some models are intrinsically more appealing than others because they require fewer arbitrary adjustments to achieve a successful fit to the data. In the immediate context of mechanisms of layer formation in layered intrusions, we show that purportedly incontrovertible evidence for in situ crystallization of magnetite can better be used to support a role for melt infiltration from below an initially homogeneous magnetite crystal mush, but the choice of model remains a matter of preference. 
51 The Upper Zone of the Bushveld Complex crystallized from an iron-rich basaltic andesite

52

53

54

55

56

57 magma at the top of the Rustenburg Layered Suite in what appears to have been a meltdominated sill hundreds of $\mathrm{km}$ wide and at least several hundred $\mathrm{m}$ deep ${ }^{17}$. The evolution of this magma and its cumulates can be modeled broadly as the product of a protracted process of fractional crystallization at the scale of the entire Upper Zone $e^{3,17,20}$, however the Upper Zone contains at least 26 magnetitite layers that are $\sim 0.1-10 \mathrm{~m}$ thick and concordant with the igneous layering. The formation of these magnetite layers cannot be accommodated by a simple process of fractional crystallization from a single homogeneous body of liquid the size of the entire Upper Zone. Instead, it seems probable that a repeated cyclic process of spontaneous double diffusive convection occurred wherein the rejected liquid complementary to the gabbroic cumulates was denser than the main body of magma, causing the system to become stratified with a dense Fe-rich residual melt separating the growing cumulate pile from the rest of the magma ${ }^{17,21}$. It is thought that when magnetite became saturated and a layer of magnetitite was crystallized from this dense Fe-rich layer the liquid density rapidly diminished, causing convective overturn and re-homogenization of the magnetite-depleted basal layer with the rest of the magma and terminating magnetite crystallization until the closing stage of the next such cycle. Magnetitite layers generally have sharp and smooth or undulating boundaries with underlying anorthosites, but grade upwards into magnetite-rich gabbro due to smoothly increasing plagioclase modal abundance ${ }^{13,17}$. Along with abundant anorthosite xenoliths, narrow plagioclase-rich layers are widely developed within magnetitite layers (Fig. 1). The other portions are composed almost entirely of magnetite with minor interstitial plagioclase and pyroxene, effectively constituting monomineralic adcumulates. The economically important Main Magnetite Layer (MML), near the bottom of the Upper Zone, has $\sim 1-2.5 \mathrm{~m}$ thickness and crops out intermittently over hundreds of $\mathrm{km}$ of strike extent $^{10-14,22}$. It has been the focus of efforts to explain magnetitite formation. 
77 Although the origin of magnetitite layers has spurred an ongoing debate between various

78 hypotheses ${ }^{22}$, there has been general agreement that the magnetitite we observe now is an

79 adcumulate evolved from a magnetite-dominated mush with a high initial amount of

80 interstitial melt ${ }^{13,23}$. However, Kruger and Latypov ${ }^{18}$ recently proposed that this mushy zone

81 had negligible thickness, being deposited directly onto a solid substrate in situ to form

82 magnetite adcumulates with little or no initial interstitial melt. The highly compatible

83 behavior of $\mathrm{Cr}$ in magnetite ${ }^{24}$ makes it sensitive to magmatic processes (e.g., crystallization

84 and reactive melt infiltration), and the quasi-exponential vertically decreasing $\mathrm{Cr}$ gradients in

85 monomineralic magnetitite layers have been widely attributed to fractional crystallization at

86 or near the magma chamber floor ${ }^{10-12,14-15,18}$. Failure of a simple one-stage Rayleigh

87 fractionation model to match observed compositional profiles (e.g., Fig. 1a-d) led to propositions that the supply of $\mathrm{Cr}$ was modulated by double-diffusive convection in overlying melt domains ${ }^{10-12,14-15}$. By repeatedly changing the $\mathrm{Cr}$ concentration in the melt, model trends

90 could be produced that matched the data. Models of this sort can be shown to be consistent

91 with observations but are not persuasive means to compare hypotheses when the boundary

92 conditions are endlessly changeable, thus ensuring that a fit to the data can always be

93 achieved. 

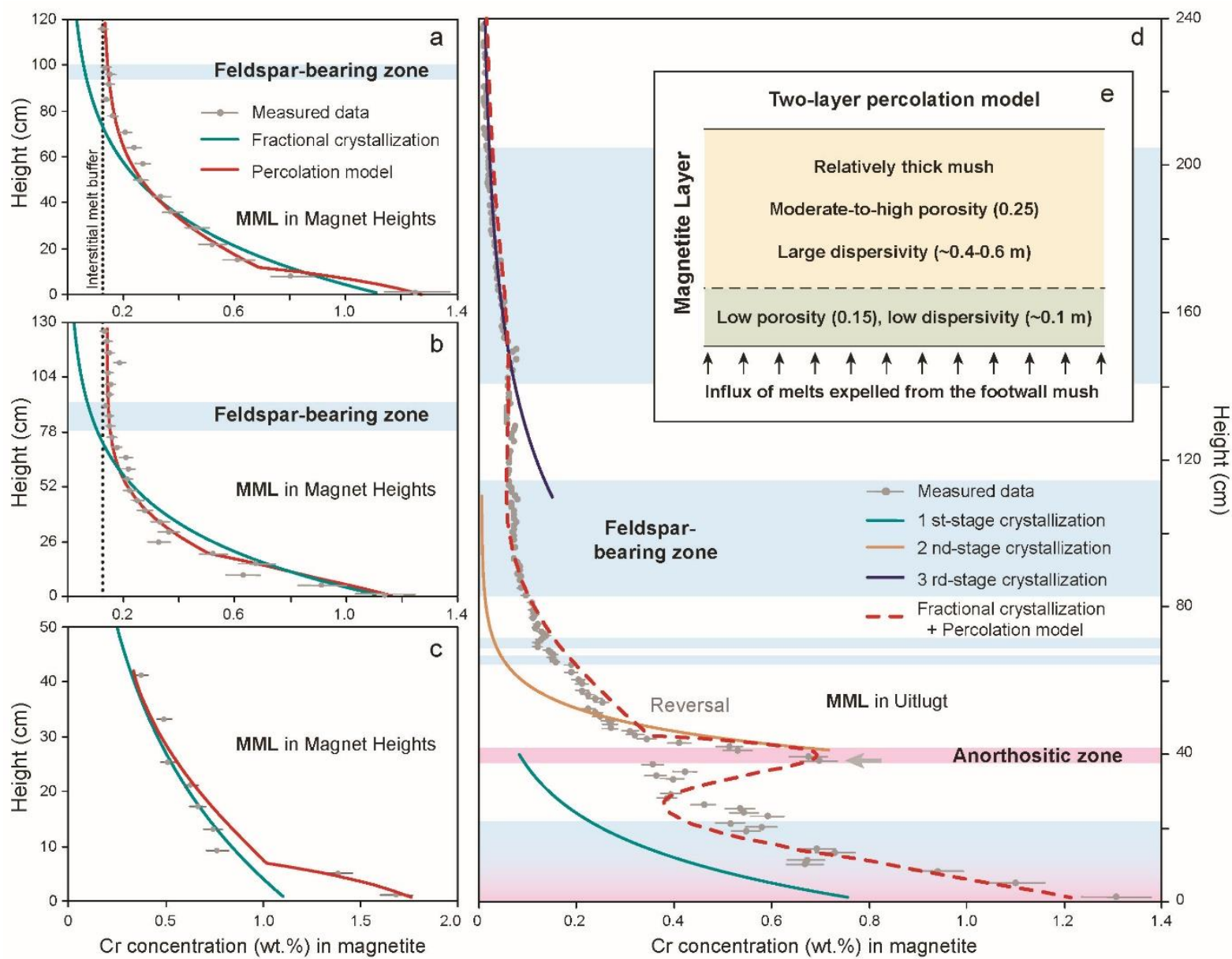

Fig. 1 (a-d) Measured and modeled vertical profiles of $\mathrm{Cr}$ contents in magnetite from the

MML. Grey arrow in (d) reflects a major reversal in $\mathrm{Cr}$ content where a new crystallizing sequence began. (e) Diagram for the two-layer percolation model. All data collected from multiple sources cited in the text, e.g., profiles in $(\mathbf{a}-\mathbf{b})^{22},(\mathbf{c})^{18}$ and $(\mathbf{d})^{12}$.

100 Results

101 Fractional crystallization models. Vertical profiles of $\mathrm{Cr}$ distribution in the MML, shown from Magnet Heights and Uitlugt in Figure 1, commonly show a curvilinear upward depletion from the bottom, which broadly matches expectations based on the compatibility of

104 Cr during fractional crystallization of magnetite ${ }^{10-12}$, but in each case the predictions do not match the data exactly. The Cr content decreases markedly more steeply at the base of every section, it locally shows extreme reversals (Fig. 1d), and it becomes constant at higher levels, 
none of which it could do during fractional crystallization unless the melt composition or basic physical parameters changed repeatedly during the process ${ }^{14-15}$. A further complicating matter is the common occurrence of exceptionally Cr-rich nodes decorating the basal contact

110 of the MML, showing strong lateral as well as vertical compositional gradients ${ }^{16,18-19}$. These

111 features have been taken as evidence that magnetite initially nucleated and crystallized

112 around discrete nodes which coalesced as they grew outwards and upwards ${ }^{16,18}$.

114 We begin by addressing the profiles at Magnet Heights, shown in Figure 1a-c. The more 115 complex Uitlugt profile will be addressed afterward. Here we assume that the MML is 116 crystallized from a thick magma sheet, and that magnetite is the sole crystalline phase during 117 the formation of the crystal mush of the MML (Methods). In Figure 1 we show models of 118 fractional crystallization of magnetite from a homogeneous body of melt containing $57 \mathrm{ppm}$

$119 \mathrm{Cr}^{3,25}$, with $f_{\mathrm{O} 2}$ between zero and one log unit below the quartz-fayalite-magnetite oxygen 120 buffer (i.e., $-1<\Delta \mathrm{QFM}<0)^{20,26-27}$ using a magnetite-melt partition coefficient of 200 at $1211150{ }^{\circ} \mathrm{C}^{14,24}$. If the initial thickness of the overlying magma is set to $120 \mathrm{~m}$ as shown (Table 122 1), on the order of the vertical distance to the next magnetite layer up section ${ }^{17,20,28}$, then the exponential decrease in $\mathrm{Cr}$ between 10 and $40 \mathrm{~cm}$ is reproduced by the model but in the upper

124 portions of all of the profiles the model predicts much greater depletion of $\mathrm{Cr}$ than is

125 observed. Furthermore, there is a short interval at the base of the MML in which Cr decreases 126 much faster than predicted by the model (Fig. 1a-c). A simple model of fractional

127 crystallization alone therefore only matches a short interval of the observed profile and is 128 inadequate. Like our predecessors ${ }^{14-15}$, we conclude that more complex processes have been 129 at play. 
131 We propose two mechanisms to account for the departures of the Magnet Hill Cr profiles

132 from fractional crystallization, both contingent on the assumption that the magnetitite

133 cumulate was initially solidified as a mush that retained some porosity occupied by interstitial

134 melt $^{13,23}$. This could be consistent either with in situ crystallization or settling of magnetite

135 crystals formed by homogeneous nucleation. First, convection of intercumulus melt at the top

136 of the pile ${ }^{29-30}$ might have promoted thorough re-equilibration of magnetite grains with the

137 overlying melt body (i.e., compositional convection) to homogenize the upper portions of the

138 pile and generate the flat profiles above $\sim 60-80 \mathrm{~cm}$ in all profiles. Second, expulsion of $\mathrm{Cr}-$

139 rich intercumulus melt from underlying anorthositic cumulates (i.e., reactive melt infiltration)

140 might have allowed upgrading of $\mathrm{Cr}$ contents in the basal parts of the layer. Both processes

141 depend on the rapid re-equilibration of magnetite crystals with percolating melt, which we

142 address below after considering the effects of compaction of the mush.

144 Compaction of magnetite mush. Isolated plagioclase crystals surrounded by magnetite

145 record weaker deformation than clustered grains, suggesting that magnetite mush contained

146 enough interstitial melt during early compaction to let the isolated crystals move or rotate

147 freely ${ }^{23}$. Poikilitic pyroxene in magnetitite commonly encloses many separate and loosely

148 packed subhedral-euhedral magnetite grains ${ }^{13}$; therefore, whether magnetite crystallized in-

149 situ or settled into place, there was initially substantial intercumulus melt. The sizes of the

150 primary grains $(\sim 0.5-2 \mathrm{~mm})$ are similar with that of disseminated magnetite in associated

151 cumulate rocks, but far less than the crystals in silicate-free regions $(\sim 5-20 \mathrm{~mm})^{13}$, indicating

152 extensive overgrowth, compaction, and annealing to form the adcumulates. If it formed by a

153 process of fractional crystallization as a loose pile of settled crystals $^{3-4}$ or as a loose

154 arrangement of chains of magnetite crystals nucleated against others in situ ${ }^{16,18-19}$ and

155 accumulated faster than the rate of compaction, the initial magnetite cumulate may have had 
156 a high porosity $(\sim 0.52)^{31}$. Compaction is driven by the large density contrast between solid $157\left(\sim 4955 \mathrm{~kg} \cdot \mathrm{m}^{-3}\right)$ and liquid $\left(\sim 2683 \mathrm{~kg} \cdot \mathrm{m}^{-3}\right)$ (Methods). Generally, this process initiates with a

158 mechanical reorganization to achieve optimum packing and continues with pressure

159 solution/reprecipitation and possible viscous deformation of cumulate minerals ${ }^{29,31-33}$. The

160 thickness of the MML (< 2.4 m) may not have provided sufficient gravitational load and 161 effective stress to drive plastic deformation of magnetite ${ }^{23}$, and hence we argue that the compaction process was likely to have been achieved mainly through pressure solution and reprecipitation. On the basis of centrifuge experiments, Manoochehri and Schmidt ${ }^{31}$

164 quantified the compaction rate for a chromite cumulate in basaltic magma via dissolution-

165 precipitation compaction. Given the affinity between chromite and magnetite, here we assume that the rate of chemical compaction for a magnetite mush layer is similar to that for chromite cumulate. The required time to reduce porosity from the initial value $(\sim 0.52$, the average porosity after crystal settling) to about 0.25 in a magnetite cumulate is in the range from 10 to 40 years, or even less if crystals are smaller (Fig. 2a; see Methods for details of 170 this calculation).

172 Compositional convection and re-equilibration. While compaction is under way, continued crystallization of magnetite from intercumulus melt locally decreases the density of

174 interstitial melt to a value lower than that of overlying magma, possibly causing it to rise as

175 buoyant plumes into the overlying melt that must be convectively replaced by melt from the 176 main magma body above the cumulate in the process of compositional convection ${ }^{29-30}$.

178 During this process, the interstitial melt is continuously expelled and replaced by the 179 overlying melt from the main magma body, maintaining a near-constant composition for the 180 pore melt ${ }^{29,34}$. The occurrence of significant compositional convection requires that the 
181 dimensionless local solutal Rayleigh number $\left(R_{a}\right)$ exceeds a critical value of about $25^{30,35}$ and

182 that the characteristic convective velocity is higher than the rate of crystal accumulation ${ }^{29}$.

183 The latter requirement is easily achieved in deep and large intrusions (e.g., the Upper Zone of

184 the Bushveld Complex) due to the small accumulation rate $\left(\sim 0.1-1 \mathrm{~m} \cdot \text { year }^{-1}\right)^{29}$. Therefore, the

185 minimum thickness of the magnetite cumulate pile to allow compositional convection to be

186 operative can be estimated when the $R_{a}$ value of porous medium equals the critical value

187 (Methods). Figure 2b shows the minimum mush layer thickness to achieve the critical

188 Rayleigh number permitting the onset of compositional convection for a range of grain sizes

189 if the decrease of melt density is driven by the crystallization of a further $2.5 \mathrm{wt} . \%$ magnetite

190 from the intercumulus melt. For example, convection begins when the thickness of an initial

$1911 \mathrm{~mm}$-grained crystal mush exceeds $\sim 0.44 \mathrm{~m}$ at $25 \%$ porosity (black dot in Fig. $2 \mathrm{~b}$ ), while a lower porosity $(15 \%)$ will require a much thicker magnetite cumulate $(\sim 2 \mathrm{~m}$, grey dot in Fig.

2b) for compositional convection. Compositional convection is therefore expected to operate

194 in the loosely packed upper portion of the mush layer but will cease once compaction has

195 reduced porosity.

197 During compositional convection and percolation, the interstitial melt will continuously modify the compositions of packed magnetite grains. Previous investigations have identified occurrences of compositionally heterogenous magnetite grains, but the observed systematic

200 zonations of $\mathrm{Al}$ and $\mathrm{Mg}$ are solely related to the exsolution of spinel phases in the sub-solidus 201 stage $^{36}$. Some fluctuations of Cr occur in the rims of magnetite grains in contact with ilmenite or plagioclase and are also attributed only to the local sub-solidus re-equilibrium ${ }^{36}$. Observed lack of other types of zonation therefore indicates that any compositional modifications of magnetite grains driven by the convection and percolation are likely to have been completed in the early stage of post-cumulate processes. Re-equilibration between convecting interstitial 
melt and magnetite grains involves two kinetic processes: the volume diffusion in single magnetite grains and the diffusion of elements through a viscous and diffusive boundary layer between the surrounding melt and magnetite (i.e., convective diffusion) ${ }^{37}$. The volume diffusion of $\mathrm{Cr}$ in a magnetite grain is simplified as the penetration diffusion of $\mathrm{Cr}$ into a spherical magnetite from an infinite source (i.e., interstitial melt) ${ }^{38-39}$ (Methods). Due to the relative motion between flowing interstitial melt and magnetite, the supply of nutrient for magnetite growth is maintained fast enough to support our assumption of a constant boundary composition on the surfaces of magnetite crystals for the volume diffusion ${ }^{40}$. Our models for magnetite growth controlled by diffusion through a narrow boundary layer against a convecting melt ${ }^{40}$ and volume diffusion within solid magnetite ${ }^{38-39}$ confirm that $\mathrm{Cr}$-poor grains (size $<1 \mathrm{~cm}$ ) rapidly approach equilibrium with passing melt, and the required time is reduced with increasing initial $\mathrm{Cr}$ contents of the grains (Fig. 2c-d). Most magnetite grains would attain local equilibrium with the percolating melt in the post-cumulus stage, except for the retention of $\mathrm{Cr}$ gradients in rare coarse crystals $(>1 \mathrm{~cm})^{36,41}$. We therefore assume that $\mathrm{Cr}$ content of magnetite in the dominant fine-grained population will be equilibrated with percolating melts.

223 Based on the $\mathrm{Cr}$ content of clinopyroxene in the closest hanging wall of the $\mathrm{MML}^{27}$, the 224 overlying magma is proposed to have contained $\sim 6.2 \mathrm{ppm} \mathrm{Cr}$ after the formation of the 225 magnetite mush. Continuous loss of buoyant interstitial melt and its replacement by $\mathrm{Cr}$ depleted melt containing $\sim 6.2 \mathrm{ppm} \mathrm{Cr}$ would maintain the upper magnetite grains at a constant Cr content ( 1240 ppm), which fits the profiles in Fig. 1a-b. Plagioclase can appear in the sequence without any correlation to the Cr trend (Fig. 1), because in this situation $\mathrm{Cr}$ content of magnetite has become decoupled from its initial value, whereas plagioclase would resist diffusive re-equilibration because solid state diffusion is so slow in feldspar ${ }^{42}$. We 

magma column from which it initially crystallized (cf. McCarthy and Cawthorn ${ }^{12}$ ).

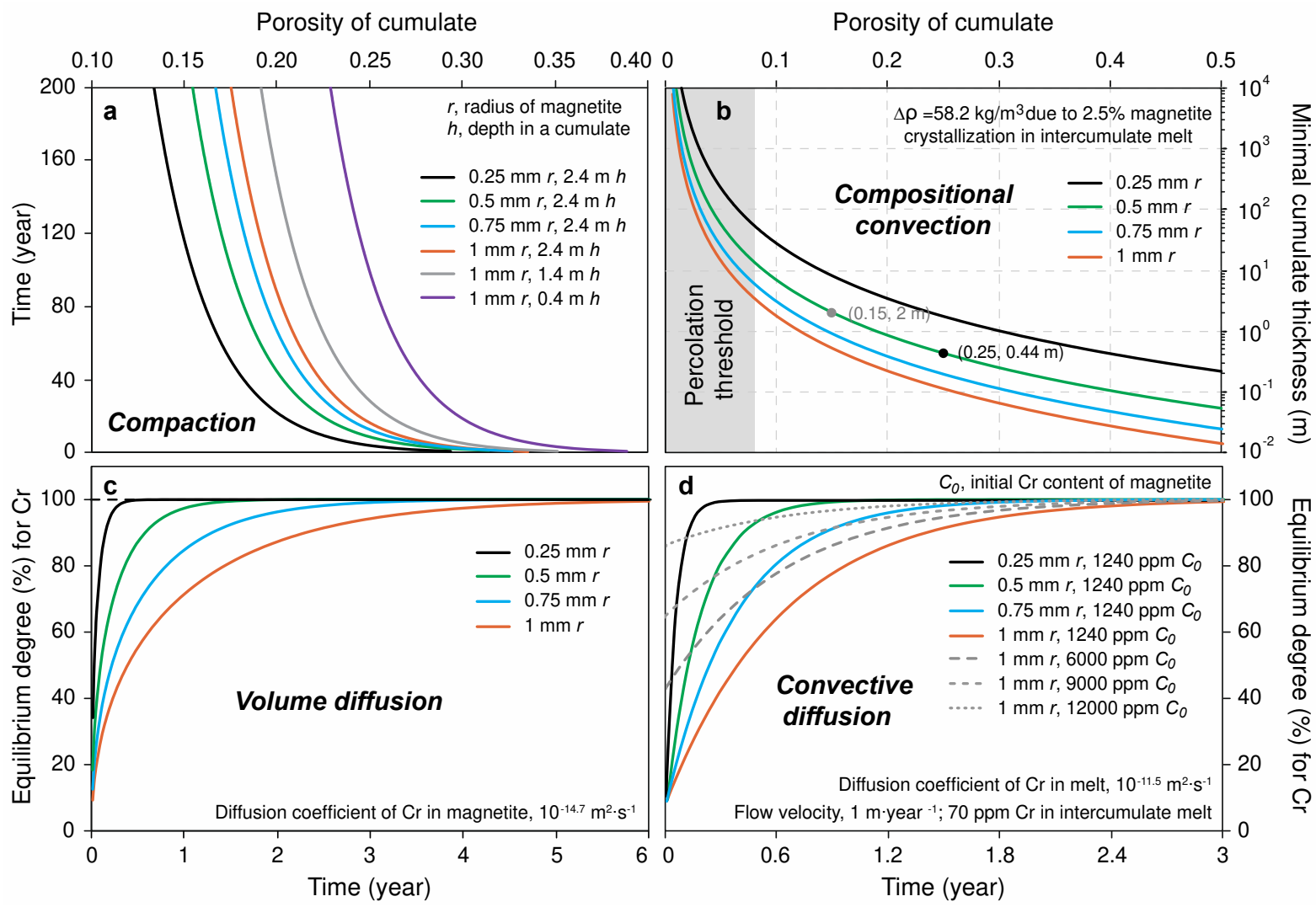

Fig. 2 Modeling results for (a) variation of magnetite mush porosity with time due to

compaction; (b) Minimum thickness allowing compositional convection for mushes with indicated porosities. More thoroughly compacted lower portions of the mush may not spontaneously initiate compositional convection whereas more open upper portions could; (c) Approach of the bulk composition of magnetite grains (integrated over the entire diffusion

240 profile) during volume diffusion of $\mathrm{Cr}$ due to re-equilibration of magnetite with migrating

241 intercumulus melt; (d) Approach of bulk composition to equilibrium via convective diffusion

242 for various sizes and initial $\mathrm{Cr}$ contents of magnetite. Coloured lines are for crystal sizes as in

$243(\mathbf{a}, \mathbf{c})$, and dashed lines represent magnetite grains with different initial Cr contents. Time

244 scales of diffusive re-equilibration are much shorter than the time scale of compaction, 
supporting the assumption of instantaneous magnetite-melt equilibrium in the percolation model.

247 Table 1 Parameters for the fractional crystallization and reactive melt infiltration models

\begin{tabular}{|c|c|c|c|c|c|c|}
\hline \multirow{2}{*}{\multicolumn{2}{|c|}{ Vertical profile }} & \multicolumn{2}{|c|}{ Fractional crystallization } & \multicolumn{3}{|c|}{ Reactive melt infiltration model } \\
\hline & & $\begin{array}{c}\text { Initial Cr in } \\
\text { magma (ppm) }\end{array}$ & $\begin{array}{c}\text { Thickness of } \\
\text { magma sheet }(\mathrm{m})\end{array}$ & $\begin{array}{c}\mathrm{Cr} \text { in } \\
\text { upwelling } \\
\text { melt (ppm) }\end{array}$ & $\begin{array}{c}\text { Initial } \mathrm{Cr} \text { in } \\
\text { magnetite }(\mathrm{ppm})\end{array}$ & $\begin{array}{l}\text { Duration } \\
\text { time (year) }\end{array}$ \\
\hline \multicolumn{2}{|c|}{ Section-a } & 57 & 120 & 73.5 & 1240 & 70 \\
\hline \multicolumn{2}{|c|}{ Section-b } & 57 & 120 & 73.5 & 1240 & 58 \\
\hline \multicolumn{2}{|c|}{ Section-c } & 57 & 120 & 108 & 1240 & 51 \\
\hline \multirow{3}{*}{ Section-d } & $1-\mathrm{st}$ & 40 & 60 & \multirow{3}{*}{70} & \multirow{3}{*}{$*$} & \multirow{3}{*}{24} \\
\hline & 2-nd & 40 & 30 & & & \\
\hline & 3-rd & 7.5 & 145 & & & \\
\hline
\end{tabular}

$248 *$ The initial Cr content of magnetite in this profile follows the variation driven by the first,

249 second, and third fractional crystallization events, and is shown as the coloured lines in

250 Figure $1 \mathrm{~d}$.

251

252 Reactive melt infiltration. The footwall of the MML is proposed to have been permeated by

253 interstitial melt that carried a slightly more primitive signature with it from lower parts of the

254 UUMZ crystal pile, causing reverse zoning of cumulus plagioclase ${ }^{21}$ and crystallization of

255 more anorthitic interstitial grains ${ }^{23}$. This slightly more primitive interstitial melt would retain

256 high Cr content because it had not yet equilibrated with magnetite in the footwall of the

257 MML, further either enhanced by crystallization of Cr-free plagioclase in the footwall

258 anorthositic mush or minimally diminished by the onset of crystallization of trace amounts of

259 intercumulus magnetite. This Cr-rich melt would be discharged upward by the compaction of

260 the footwall mush ${ }^{28}$. Hence, we presume an upward percolation of $\mathrm{Cr}$-rich melt into the base

261 of the recently formed magnetite mush, causing continuous modification of the $\mathrm{Cr}$

262 concentrations of magnetite grains. 
264 We solved this reactive transport problem using the Brinkman equations coupled with

consideration of the dispersion around magnetite crystals via COMSOL Multiphysics for a two-dimensional system with a horizontal magnetite layer sitting above an anorthositic layer (Methods). The variation of porosity in the compacting magnetite cumulate as shown in Figure $2 \mathrm{a}$ is simplified as two-layer model: a thin bottom zone $(10 \mathrm{~cm})$ with low porosity is overlain by thick mush with a higher porosity (Fig. 1e). In our model, a slightly Cr-enriched interstitial melt ( 70-74 ppm Cr for the profiles in Figs. 1a-b, d; Table 1) is expelled from underlying anorthositic mush at a compaction-driven velocity of $\sim 1 \mathrm{~m} \cdot$ year $^{-1}{ }^{29,32-33}$. The basal magnetite from the section in Figure 1c contains a larger $\mathrm{Cr}$ content $(\sim 1.7$ wt.\%) and, accordingly, the magnetite-saturated melt expelled from footwall mush is assumed to have had a higher $\mathrm{Cr}$ content ( $108 \mathrm{ppm}$, Table 1$)$, which is equal to the $\mathrm{Cr}$ amount of the intruding parental magma above the Pyroxenite Marker ${ }^{43}$.

As a simple end-member scenario, the red lines in Figure 1a-c show the results of the percolation model imposed on a pile of magnetite crystals along a one-dimensional profile through the top of one of the nodes in the two-dimensional model described below (Fig. 2). To identify better the influence of the percolation process, the magnetite grains in the whole cumulate pile are assumed initially to be equilibrium with a Cr-depleted, evolved melt $(\sim 6.2$ ppm Cr, Table 1), i.e., they initially contain a constant $\mathrm{Cr}$ content ( $1240 \mathrm{ppm})$ in the whole profile due to the effects of the compositional convection described above. Our percolation model trends account well for the steepening of $\mathrm{Cr}$ compositional gradients at the bottoms of all four one-dimensional sequences documented in Figure 1a-d, and the flat upper portions remain as imposed by reaction with the overlying melt due to compositional convection within the mush. 
289 Lateral variations in Cr concentration. Others have inferred that concentration contours

290 concentric around node-like structures at the base of the MML were caused by in situ growth

291 that was initiated at discrete points on the floor ${ }^{16,18-19}$. Instead, we used our two-dimensional

292 model of percolative reactive transport to address the alternative hypothesis that local

293 variations in porosity in the underlying anorthosite mush may allow some sites to become

294 preferred pathways for underlying melt during upward percolation. If Cr-rich melts flow

295 through these nodes with a higher velocity and greater net mass flux, the grains here are able

296 to extract more $\mathrm{Cr}$ than surrounding crystals, inducing lateral variations in $\mathrm{Cr}$ content along

297 the base (Fig. 3a). Along with melt dispersion and upward flow in mush, this effect extends

298 outwards to form undulations in Cr-concentration contours which gradually weaken over

$299 \sim 20-30 \mathrm{~cm}$ height (Fig. 3b-c), coinciding with the geochemical mapping on field outcrops of

300 the $\mathrm{MML}^{18}$. We included an anorthosite autolith in the basal mush and considered that a solid

301 block may impede early compaction of magnetite, leaving a high-porosity domain around the

302 block which would enhance percolation. The resulting perturbation in the flow field led to

303 concentric Cr enrichment around this autolith (Fig. 3d), producing spatial variations very

304 similar to those shown by Kruger and Latypov ${ }^{18-19}$. This also partly accounts for the

305 confusing phenomenon that Cr-rich growth nodes are found in the nooks beneath the

306 autoliths but not directly on the upper surfaces of autoliths ${ }^{18-19}$. 


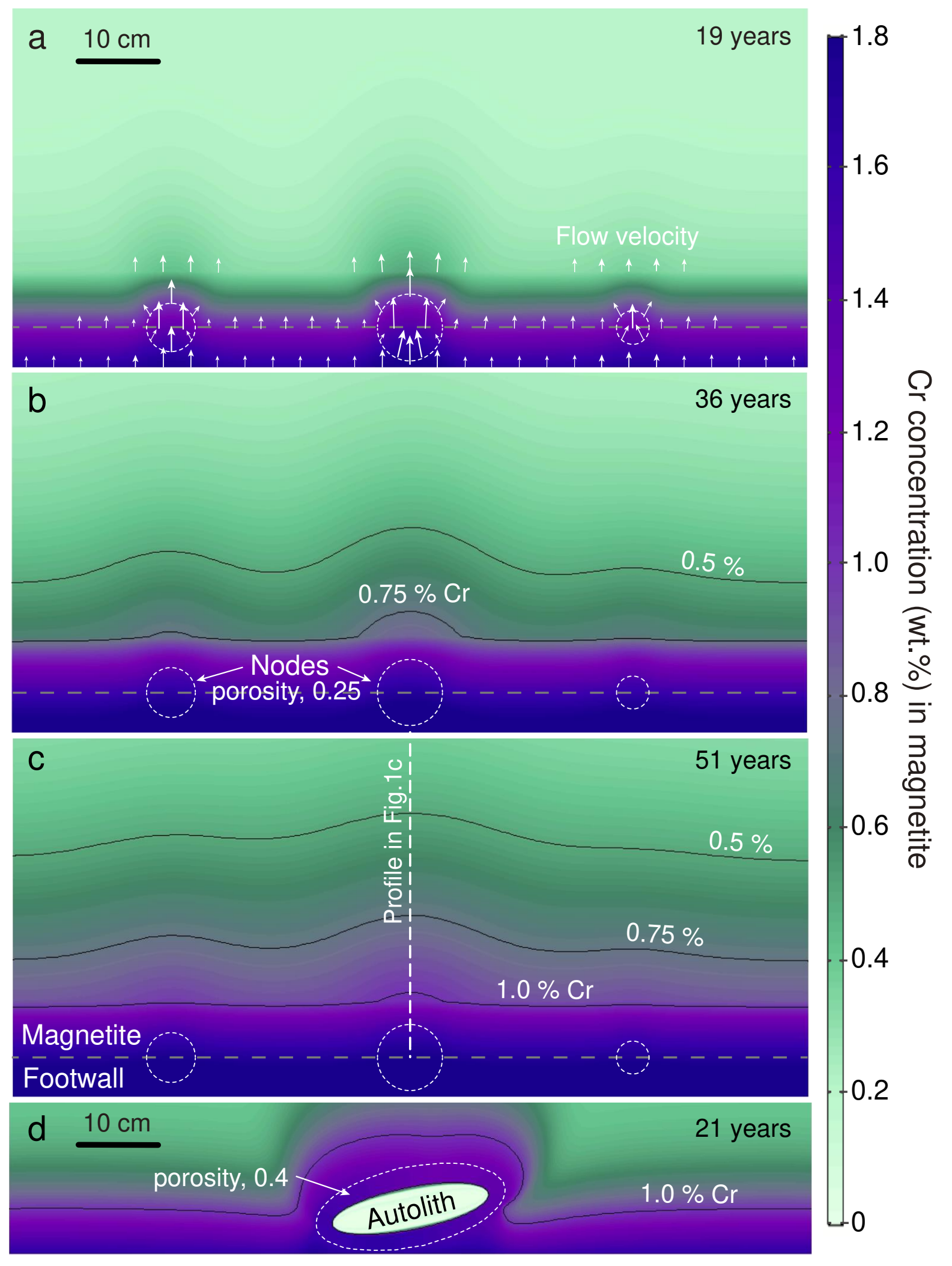

Fig. 3 (a-c) Snapshots of modeled Cr compositional mapping for the percolation of basal

309 magnetite mush with three high-porosity spheric nodes $(0.25$, white dashed lines). White

310 arrows in (a) plot for melt flow vectors. (d) Cr contours drape over the anorthosite autolith 
due its high-porosity contact zone (0.4, white dashed line) with magnetite mush. Black lines represent $\mathrm{Cr}$ isopleths.

313

314 Multiple episodes of fractional crystallization combined with reactive transport. More

315 complex profiles than those at Magnet Heights (Fig. 1a-c) require more complex adjustments

316 to boundary conditions. We have combined the fractional crystallization model with the

317 reactive transport model to address the complexity of the Uitlugt profile, which was sampled

318 at $1 \mathrm{~cm}$ spacings. The large excursion in $\mathrm{Cr}$ content at $40 \mathrm{~cm}$ shown in Figure $1 \mathrm{~d}$, which

319 contains about $65 \%$ plagioclase, was reported not to be present in flanking boreholes by

320 McCarthy and Cawthorn (1983), who attributed it to unspecified local convective

321 fluctuations in magma composition during magnetite crystallization. To account for the evident presence of three segments of the profile, each decaying from progressively lower starting points, we accept the possibility that convective overturn after deposition of the first

$324 \sim 40 \mathrm{~cm}$ of magnetite led to the start of a new episode of fractional crystallization from fresh

325 Cr-rich melt, and a third such event occurred after deposition of about $110 \mathrm{~cm}$ of magnetite,

326 however we do not resort to a change in magma composition every time the profile departs

327 from a perfect exponential decay. In Figure 1d we show the three initial profiles of $\mathrm{Cr}$ due

328 purely to fractional crystallization, as well as the trend resulting from 24 years of continued

329 evolution of $\mathrm{Cr}$ due to percolative reactive transport of $\mathrm{Cr}$-rich intercumulus melt from below

330 (Table 1). The combined fractional crystallization and reactive transport model duplicates all

331 the observed peaks and inflections in slope of Cr variation, using only three discrete

332 crystallization sequences, for each of which the only adjustable parameter was the initial $\mathrm{Cr}$

333 content of the new magma. 
336 The difference between models of in situ magnetite crystallization and collection of loose

337 crystals of magnetite may seem like a moot point, but it drives at the difference between two

338 fundamentally different mechanisms of layer formation. If crystals in layered intrusions form

339 within the melt and are transported to their eventual resting places, the implications for

340 petrogenesis are dramatically different than they are if crystals form in situ on a hard

341 substrate. A very wide range of possibilities is consistent with deposition of a mush, but only

342 one is permitted if crystallization proceeded in situ. It should be evident at this point that the

343 match between observations and models afforded both by our models and by the previously

344 published models places one in the uncomfortable position of having to choose between two

345 very different sets of mechanisms to explain exactly the same observations with equal

346 fidelity. In summary, all existing models including ours rely fundamentally on the idea that

347 fractional crystallization of magnetite has generated the rapid and approximately exponential

348 decay of $\mathrm{Cr}$ concentration in vertical profiles. The models differ in their explanations of

349 inflections or reversals in the $\mathrm{Cr}$ profiles (Fig. 1) and of perturbations of $\mathrm{Cr}$ concentration

350 contours due to lateral variations (Fig. 3).

351

352 Previous workers have inferred that the deposition of magnetite occurred in situ along the

353 base of the magma chamber ${ }^{10-12,16,18}$. In these models there was no significant role for trapped

354 liquid between magnetite crystals as they formed; lateral variations in $\mathrm{Cr}$ content were

355 accounted for as resulting from initial magnetite nucleation at isolated growth centres that

356 grew concentrically until they merged, and inflections in slope of $\mathrm{Cr}$ concentration in vertical

357 profiles were accounted for by numerous adjustments to the boundary conditions ${ }^{16,18}$. These

358 adjustments varied from one model to the next, but all were designed to control the supply of

359 Cr to the fractionating magnetite. In our models, magnetite collected as discrete crystals to

360 form an orthocumulate-textured mush during fractional crystallization; reversals of $\mathrm{Cr}$ 
gradients in more complex profiles are accounted for by as many as three episodes of

362

363

364

365

366

367

368

370

371 Geological observations can impose the primary criteria for testing these forward models.

372 First, we consider whether it is more likely that magnetite arrived in its present location by in

373

374

375

376

377

378 convective overturn in the overlying magma (Fig. 1d). Percolation of melt through the mush could both have erased early compositional gradients in the upper portions of thick mushes and might also have allowed upward-migrating intercumulus liquid from below to steepen the $\mathrm{Cr}$ concentration gradients near the base. Lateral variations in $\mathrm{Cr}$ concentration are accounted for by postulating that the porosity of the underlying anorthosite mush was not uniform; randomly distributed local enhancements in anorthosite permeability drove increased percolation at discrete locations in the base of the mush, generating concentricallyzoned domical patches of Cr enrichment (Fig. 3).

\section{First, we consider whether it is more likely that magnetite arrived in its present location by in} situ growth due to heterogeneous nucleation on a hard floor or by homogeneous nucleation in the melt followed by crystal settling to form a mush. The presence of plagioclase with magnetite in continuously variable proportions along with the abundance of autoliths is suggestive of co-accumulation of discrete grains due to crystal settling. The preservation of considerable relict intercumulus volume in pyroxene-poikilitic domains ${ }^{13}$ indicates that whether it occurred heterogeneously or homogeneously, magnetite crystallization initially led to formation of an open framework through which intercumulus melt would have been free to migrate before compaction and/or adcumulus growth brought porosity down below the percolation threshold (Fig. 2b) ) $^{29,44}$. In-situ crystallization experiments at low undercooling document the homogeneous nucleation of magnetite in basaltic melt even after initial heterogeneous nucleation on a solid substrate ${ }^{45}$. During diffusion-controlled growth, the preferred sites of incipient nucleation and rapid growth of magnetite would be expected to lie at the highest points on the surface rather than in deep reentrants under autoliths, regardless 
386 of their origins as relicts of eroded floor rocks ${ }^{19}$ or as xenoliths. Taken all together, these

387 observations and interpretations alone do not allow a firm choice between homogeneous

388 nucleation followed by crystal settling, or heterogeneous nucleation in situ at the magma

389 chamber floor, but we lean towards a process dominated by homogeneous nucleation from

390 the melt followed by settling of magnetite to form a highly permeable initial cumulate

391 framework.

392

393 The choices of model parameters and boundary conditions may reflect the robustness and

394 reliability of these forward models. Previous models resorted to diffusive addition of $\mathrm{Cr}$ from

395 convecting layers above $\mathrm{e}^{10-12,14-15,46}$. Considering diffusive supply from a reservoir of fixed

396 size to the crystallizing magnetite Wright et al. ${ }^{46}$ and McCarthy et al. ${ }^{14}$ found that, to fit the

397 measured profiles for a fixed value of the $\mathrm{Cr}$ diffusion coefficient $\mathrm{D}$ in the melt, the rate of

398 crystal accumulation had to be arbitrarily constrained by the observed compositions. The rate

399 of crystal accumulation implied by the model would require as much as $3 \mathrm{Myr}$ to crystallize

400 the $1 \mathrm{~m}$ thick MML ${ }^{15}$. Alternatively, Kruger and Smart $^{15}$ showed that the profile could be

401 fitted very well by assuming a continuous influx of $\mathrm{Cr}$ into the crystallizing layer of magma

402 from an upper layer, above a stable diffusive boundary layer, in a stratified magma

403 undergoing double diffusive convection driven by crystallization at the base and heat loss out

404 the top. However, their model could only be made to fit the observed compositional profiles

405 by adopting extremely high and unrealistic values of $D_{C r}$ of about $10^{-9} \mathrm{~m}^{2} \cdot \mathrm{s}^{-1}$ whereas

406 measured values in comparable andesitic melts are about 300 times smaller $\left(3.2 \times 10^{-12} \mathrm{~m}^{2} \cdot \mathrm{s}^{-1}\right.$

407 at $\left.1150{ }^{\circ} \mathrm{C}\right)^{47}$. It is possible that by changing other model parameters their curves could be

408 brought into line with measured data. The parameter least well constrained in their model is

409 the thickness of the diffusive boundary layer separating the crystallizing lower layer from the

410 layer above it. Since they never specified the value that they chose for the boundary layer 
thickness it is impossible to judge whether or not it could accommodate a change sufficient to

412 match the data using correct values for $D_{C r}$. In any case, both of these previous models work

413 only if a key model parameter such as the thickness of a hypothetical diffusive boundary

414 layer can be adjusted at will. Evidently, without independent evidence to guide the choices of

415 such parameters, they can be dialed in to match any profile and the modeling process

416 becomes an arbitrary exercise in curve fitting.

417

418 Echoing the suggestion of Cawthorn ${ }^{16}$, Kruger and Latypov ${ }^{18}$ modeled solidification of the

419 MML by in situ crystallization of magnetite directly attached to a solid substrate in a process

420 of fractional crystallization from a well-mixed convecting reservoir of melt. As in other examples, their model would predict a continuous exponential decrease in $\mathrm{Cr}$ concentration and would fail to match the observed flattening of compositional profiles after the initial rapid decline (Fig. 1). To deal with this issue they added batches of fresh melt to their magma reservoir in amounts and with $\mathrm{Cr}$ contents arbitrarily chosen to force the model $\mathrm{Cr}$ trend to

425 follow the observed trend. For example, Kruger and Latypov ${ }^{18}$ required successive injections 426 of four discrete magma pules with variable volumes to account for only the lowest 9

427 measurements in a 40-cm-thick profile (Fig. 1c). When a new pulse of magma is required in a 428 model to account for every second data point, the model again becomes an arbitrary exercise 429 in curve-fitting that loses predictive explanatory power. Furthermore, the two-dimensional 430 model of magnetite crystallization had to use measured $\mathrm{Cr}$ concentration contours to guide 431 their modeled crystallization sequence, rather than having the crystallization pattern governed 432 by any physical model of diffusion-mediated crystal growth in a diffusive boundary layer.

434 There are weaknesses in our models as well. We have chosen boundary conditions and values 435 for parameters like diffusivities and partition coefficients to match external constraints as 
well as we can; however, to match the complex $\mathrm{Cr}$ concentration profile at Uitlugt we must

437 resort to three separate magma recharge events timed to generate the observed inflections and

438 we must perforce select a Cr content of the incoming melt to match the overall pattern. The

439 principal differences between our models and previous ones are that we minimized the

440 number of adjustable parameters (e.g., at most three magma influxes rather than one for every

441 two data points, model melt compositions fixed by equilibrium with measured mineral

442 compositions, use of experimentally measured compaction rates and diffusion coefficients)

443 and we accounted for processes widely thought to have occurred in the Upper Zone such as

444 post-cumulus melt migration and infiltration ${ }^{23,28,48}$ which were overlooked by previous

445 approaches.

446

\section{Conclusion and implications}

448 Forward models of magmatic processes that cannot be directly observed can be valuable as

449 descriptions of what could plausibly have happened, but they cannot be regarded uncritically

450 as demonstrations of what really happened. When we are faced with multiple contradictory models that can adequately generate an acceptable quantitative match to observations, we must make our final choice based on the law of parsimony, also called Occam's Razor - we favor the simplest hypothesis that requires the smallest number of artificially imposed external constraints while simultaneously acknowledging that we cannot be sure of the answer. A modeling scenario that can be set up to match the observations by setting nothing more than a plausible set of initial conditions and acceptable parameter choices is greatly preferable to another that requires continuous and arbitrary fine-tuning of model parameters to achieve a match to observations, even more so when the arbitrary models can only succeed through the adoption of physical parameters that are far outside of the ranges dictated by experimental observations. 
462 In the balance, although both sets of models can be shown to work to explain the data, we prefer our model of melt migration and reaction with a magnetite crystal mush that was itself

464 generated in some localities by multiple pulses of deposition from a magma sheet undergoing episodic replenishments due to convective overturn. The significance of the conclusion is that it speaks to a fundamental bifurcation in our understanding of the genesis of layered intrusions - do crystals form in situ on solid substrates or are they formed on one place and deposited in another by transport and settling? These two concepts have dramatically important effects in controlling how we understand the formation of layered intrusions in general and, more specifically, the genesis of critically important mineral deposits of $\mathrm{Fe}, \mathrm{Ti}$,

$471 \mathrm{~V}, \mathrm{Ni}, \mathrm{Cu}, \mathrm{Co}, \mathrm{Pt}, \mathrm{Pd}$ etc. The importance of in situ crystal growth is not unequivocally established by observations of the MML. Evidently our current knowledge of the Main Magnetite Layer does not, as yet, carry the information we need to make a definitive choice, and it is important for us to remember that as we continue to search for more definitive answers to a perplexing problem at the heart of igneous petrology.

477 Method

478 Equations for fractional crystallization model. In each increment of the fractional

479 crystallization, the conservation of $\mathrm{Cr}$ mass in a closed system requires that:

$$
C_{i-1}^{m e l t} \cdot H_{i-1} \cdot \rho_{i-1}^{m e l t}=C_{i}^{m e l t} \cdot k_{D}^{m t} \cdot\left(H_{i-1}-H_{i}\right) \cdot \rho_{m t}+C_{i}^{m t} \cdot H_{i} \cdot \rho_{i}^{m e l t}
$$

481 where $C_{i}^{\text {melt }}, H_{i}$ and $\rho_{i}^{\text {melt }}$ are the Cr content, thickness and density of melt at $i$-th increment, 482 respectively, and the $k_{D}^{m t}$ is the partition coefficient of Cr between magnetite and melt, and 483 here is assumed as 200 based on recent experimental measurements ${ }^{24}$. The density of magnetite $\left(\rho_{m t}\right)$ is estimated as $\sim 4955 \mathrm{~kg} \cdot \mathrm{m}^{-3} 49$. The melt density keeps decreasing due to the crystallization of denser magnetite phase, and if we make the simplifying assumption that 
the partial molar volume of the $\mathrm{Fe}_{3} \mathrm{O}_{4}$ component in the melt is the same as that of magnetite,

487 its value at the $i$-th increment can be calculated as:

488

$$
\rho_{i}^{\text {melt }}=\frac{\rho_{0} \cdot H_{0}-\rho_{m t} \cdot\left(H_{0}-H_{i}\right)}{H_{i}}
$$

489

490

491

493

494

495

496

497

498

499

500

501

502

503

504

505

506

507

508

where $\rho_{0}$ is the density of initial melt $\left(\sim 2683 \mathrm{~kg} \cdot \mathrm{m}^{-3}\right)$, and $H_{0}$ is the initial thickness of melt.

The thickness of the magnetite layer at the $i$-th increment is expressed as $\left(H_{0}-H_{i}\right)$, and the $\mathrm{Cr}$ concentration of crystallized magnetite within this increment $\left(C_{i}^{m t}\right)$ is calculated as:

$$
C_{i}^{m t}=C_{i}^{m e l t} \cdot k_{D}^{m t}
$$

Compaction time calculation. The compaction time $(t)$ is the required time to reduce porosity from the initial porosity $(\sim 0.52)$ to a given lower value in the basal layer of a magnetite cumulate, and can be calculated $\mathrm{as}^{31}$ :

$$
t=\frac{5.471 \times 10^{18}}{10^{(14.35 \cdot \varphi)}} \times \frac{d}{h \cdot \Delta \rho}
$$

where is $\varphi$ the porosity of cumulate pile, $d$ is the diameter of magnetite grains (m), $h$ is the depth in crystal mush (m), and $\Delta \rho$ is the density difference between the magnetite and interstitial melt $\left(\mathrm{kg} \cdot \mathrm{m}^{-3}\right)$. On the basis of our fractional crystallization model ${ }^{3}$, the density of the evolved melt that corresponds to the position of the MML in the Upper Zone can be estimated as $\sim 2683 \mathrm{~kg} \cdot \mathrm{m}^{-3}$ via the parameterized equation from Lesher $\& \mathrm{Spera}^{50}$.

Compositional convection model. Based on the critical value of the Rayleigh number $\left(R_{a}\right.$, $\sim 25)^{30,35}$, the minimum thickness $\left(h_{\min }\right)$ of the magnetite cumulate pile allowing compositional convection to be operative can be estimated as $^{29}$ :

$$
h_{\min }=\frac{R_{a} \cdot \mu_{m} \cdot D}{k \cdot g \cdot \Delta \rho^{\prime}}
$$

where $\mu_{m}$ is the melt viscosity $(\sim 160 \mathrm{~Pa} \cdot \mathrm{s})^{3,51}, D$ is the effective chemical diffusivity in the melt, $k$ is the permeability of crystal mush, $g$ is the gravitational acceleration, and $\Delta \rho^{\prime}$ is the density difference between the interstitial melt and overlaying magma. The diffusion 
509 coefficient of $\mathrm{Cr}$ in melt is estimated as $3.2 \times 10^{-12} \mathrm{~m}^{2} \cdot \mathrm{s}^{-147}$, and adopted as the $D$ here. The

510 decrease of melt density driven by the crystallization of $2.5 \mathrm{wt} . \%$ magnetite from the pore

511 melt is calculated as $58.2 \mathrm{~kg} \cdot \mathrm{m}^{-3}$, which can be considered as the $\Delta \rho^{\prime}$. At a high porosity, the

512 permeability of crystal mush is simplified as ${ }^{33,52-53}$ :

$$
k=d^{2} \cdot \varphi^{3} / 300
$$

514 where $d$ is the diameter of magnetite grains and $\varphi$ is the porosity of crystal mush.

515 Equation for the diffusion model. For the bulk diffusion of $\mathrm{Cr}$ in a spherical magnetite

516 grain with uniform initial composition and a boundary set to a different fixed composition by

517 reaction with an infinite surrounding medium, the equilibrium degree of $\mathrm{Cr}$ can be expressed

518 as the ratio of $M_{t}$ (total amount of diffusing Cr entering or leaving the magnetite at time $t$ ) to

$519 M_{\infty}$ (corresponds the amount of diffusing $\mathrm{Cr}$ after infinite time to reach equilibrium) $)^{38}$ :

$$
\frac{M_{t}}{M_{\infty}}=1-\frac{6}{\pi^{2}} \sum_{n=1}^{\infty} \frac{1}{n^{2}} \cdot e^{-D_{C r-m t} \cdot n^{2} \cdot \pi^{2} t / r^{2}}
$$

where $r$ is the radius of magnetite grain, $t$ is the time and $D_{C r-m t}$ is the diffusion coefficient of

$522 \mathrm{Cr}$ in magnetite $\left(\sim 2 \times 10^{-15} \mathrm{~m}^{2} \cdot \mathrm{s}^{-1}\right)^{24,37}$. On the other hand, during the convective diffusion, a

523 compositional gradient of $\mathrm{Cr}$ persists within a thin diffusive boundary layer around the

524 magnetite grain, and the mass transfer $\left(d M_{C r}\right)$ of an element $\mathrm{Cr}$ via this compositional

525 boundary layer within a short time interval $(d t)$ can be approximated as ${ }^{40}$ :

526

$$
\frac{d M_{C r}}{d t}=4 \pi r^{2} \rho_{m} \cdot D_{C r-m e l t} \cdot \frac{C_{m}-C_{0}}{\delta_{C r}}
$$

527 where $r$ is the magnetite radius, $\rho_{m}$ is the density of melt $\left(\sim 2683 \mathrm{~kg} \cdot \mathrm{m}^{-3}\right), D_{C r \text {-melt }}$ is the

528 diffusion coefficient of $\mathrm{Cr}$ in melt $\left(\sim 3.2 \times 10^{-12} \mathrm{~m}^{2} \cdot \mathrm{s}^{-1}\right)^{47}, C_{m}$ is the $\mathrm{Cr}$ content of interstitial

529 melt, $C_{0}$ is the initial $\mathrm{Cr}$ concentration of magnetite, and $\delta_{C r}$ is the boundary layer thickness

530 for $\mathrm{Cr}$. The $\mathrm{Cr}$ flux predicted by this equation is summed for successive time intervals to 


$$
\delta_{C r}=2 r /\left[1+\left(1+\frac{2 r V}{D_{C r-m e l t}}\right)^{1 / 3}\right]
$$

534 where $V$ is the flow rate of interstitial melt $\left(\sim 1 \mathrm{~m} \cdot \text { year }^{-1}\right)^{29,32-33}$.

535 Reactive melt infiltration model. The Brinkman equations extend Darcy's law to model the

536 dissipation of kinetic energy by viscous shear, which is similar to the Navier-Stokes

537 equations, and are also connected to a set of reaction-diffusion-convection equations. Here, the Brinkman equations for steady state flow are:

$$
\left\{-\nabla \cdot \frac{\mu}{\varphi}\left[\nabla \boldsymbol{u}+(\nabla \boldsymbol{u})^{T}\right]\right\}-\left(\frac{\mu}{k} \cdot \boldsymbol{u}+\nabla p-\boldsymbol{F}\right)=0 \text { and } \nabla \cdot \boldsymbol{u}=0
$$

where $\mu$ is melt viscosity, $\boldsymbol{u}$ represents the velocity vector, $p$ is pressure, $\varphi$ is the porosity and $k$ denotes the permeability of crystal mush. Gravity is also included in this model, and its influence is accounted for by the force term, $\boldsymbol{F}$. The grain size of magnetite is assumed as 2 $\mathrm{mm}$ in the mode $^{13}$, and the relation between porosity and permeability follows the Kozeny-

544 Carman model ${ }^{55}$. The transport of solute $\mathrm{Cr}$ in the migrating melt due to liquid dispersion, advection and molecular diffusion is modeled via the advection-dispersion equation:

$$
\varphi \frac{\partial \gamma}{\partial t}+\nabla \cdot\left(-\varphi \cdot D_{L} \cdot \nabla \gamma+\boldsymbol{u} \gamma\right)=S_{C}
$$

where $\varphi$ is the porosity, $\gamma$ is the mass concentration of $\mathrm{Cr}$ in melt $\left(\mathrm{kg} \cdot \mathrm{m}^{-3}\right), \boldsymbol{u}$ is the flow velocity $\left(\mathrm{m} \cdot \mathrm{s}^{-1}\right), D_{L}$ is the hydrodynamic dispersion tensor $\left(\mathrm{m}^{2} \cdot \mathrm{s}^{-1}\right)$, and $S_{C}$ represents the quantity of $\mathrm{Cr}$ added per unit volume of porous medium per unit time at the bottom of magnetite cumulate pile $\left(\mathrm{kg} \cdot \mathrm{m}^{-3} \cdot \mathrm{s}^{-1}\right)$. The hydrodynamic dispersion tensor $\left(D_{L}\right)$ describes the combined influences of the chemical diffusivity in the melt and the mechanical dispersivity via grain-scale flow in porous media. Hence, the diagonal components of the $D_{L}$ are controlled by the diffusion coefficient of $\mathrm{Cr}$ in the andesitic melt $\left(\sim 3.2 \times 10^{-12} \mathrm{~m}^{2} \cdot \mathrm{s}^{-1}\right)^{47}$, and 
the dispersivities along the longitudinal and transverse flows. Previous work demonstrated a systematic increase of the longitudinal dispersivity with the corresponding observational scale of porous media ${ }^{56}$, and we assume $\sim 0.1$ and $\sim 0.4-0.6 \mathrm{~m}$ for the two layers in modeled mush, respectively (Fig. 1e). The transverse dispersivity in experiments is typically an order of magnitude smaller than that of longitudinal dispersivity ${ }^{57}$, which is also adopted here.

\section{Data availability:}

All data shown here was obtained from the cited references.

\section{References:}

1. Namur, O. et al. Igneous layering in basaltic magma chambers. In: Chalier, B., Namur, O., Latypov, R., and Tegner, C. (eds) Layered Intrusions, 1st edn. Springer, pp. 75-152 (2015).

2. Mungall, J. E., Kamo, S. L. \& McQuade, S. U-Pb geochronology documents out-ofsequence emplacement of ultramafic layers in the Bushveld Igneous Complex of South Africa. Nat. Commun. 7, 13385 (2016).

3. Yao, Z. S., Mungall, J. E. \& Jenkins, M. C. The Rustenburg Layered Suite formed as a stack of mush with transient magma chambers. Nat. Commun. 12, 505 (2021).

4. Latypov, R., Chistyakova, S. \& Mukherjee, R. A novel hypothesis for origin of massive chromitites in the Bushveld Igneous Complex. J. Petrol. 58, 1899-1940 (2017).

5. Latypov, R., Chistyakova, S., Namur, O. \& Barnes, S. Dynamics of evolving magma chambers: textural and chemical evolution of cumulates at the arrival of new liquidus phases. Earth-Sci. Rev. 210, 103388 (2020).

6. Boudreau, A. E. \& McCallum, I. S. Concentration of platinum-group elements by magmatic fluids in layered intrusions. Econ. Geol. 87, 1830-1848 (1992).

7. Mungall, J. E. Kinetic controls on the partitioning of trace elements between silicate and sulfide liquids. J. Petrol. 43, 749-768 (2002).

8. Mungall, J. E. \& Naldrett, A. J. Ore deposits of the platinum-group elements. Elements 4, 253-258 (2008).

9. Wager, L. R. \& Brown, G. M. Layered igneous rocks. Oliver \& Boyd, Edinburgh London, 
10. Cawthorn, R. G. \& McCarthy, T. S. Variations in Cr content of magnetite from the Upper Zone of the Bushveld Complex - Evidence for heterogeneity and convection currents in magma chambers. Earth Planet. Sci. Lett. 46, 335-343 (1980).

588

589

590

591

592

593

594

595

596

597

598

599

600

601

602

603

604

605

606

607

608

609

610

611

612

613

614

615

616

617

11. Cawthorn, R. G. \& McCarthy, T. S. Bottom crystallization and diffusion control in layered complexes: Evidence from $\mathrm{Cr}$ distribution in magnetite from the Bushveld Complex. Trans. Geol. Soc. S. Afr. 84, 41-50 (1981).

12. McCarthy, T. S. \& Cawthorn, R. G. The geochemistry of vanadiferous magnetite in the Bushveld Complex: Implications for crystallization mechanisms in layered complexes. Miner. Depos. 18, 505-518 (1983).

13. Reynolds, I. M. The nature and origin of titaniferous magnetite-rich layers in the Upper Zone of the Bushveld Complex: A review and synthesis. Econ. Geol. 80, 1089-1108 (1985).

14. McCarthy, T. S., Cawthorn, R. G., Wright, C. J. \& Mclver, J. R. Mineral layering in the Bushveld Complex: Implications of $\mathrm{Cr}$ abundances in magnetite from closely spaced magnetitite and intervening silicate-rich layers. Econ. Geol. 80, 1062-1074 (1985).

15. Kruger, F. J. \& Smart, R. Diffusion of trace elements during bottom crystallization of double-diffusive convection systems: the magnetitite layers of the Bushveld Complex. J. Volcanol. Geotherm. Res. 34, 133-142 (1987).

16. Cawthorn, R. G. Growth nodes at the base of magnetitite layers in the Upper Zone of the Bushveld Complex. S. Afr. J. Geol. 97, 455-461 (1994).

17. Tegner, C., Cawthorn, R.G. \& Kruger, F. J. Cyclicity in the Main and Upper Zone of the Bushveld Complex, South Africa: crystallization from a zoned magma sheet. J. Petrol. 47, 2257-2279 (2006).

18. Kruger, W. \& Latypov, R. Fossilized solidification fronts in the Bushveld Complex argue for liquid-dominated magmatic systems. Nat. Commun. 11, 2909 (2020).

19. Kruger, W. \& Latypov, R. Magmatic karst reveals dynamics of crystallization and differentiation in basaltic magma chambers. Sci. Rep. 11, 7341 (2021).

20. Vantongeren, J. A., Mathez, E. A. \& Kelemen, P. B. A felsic end to Bushveld differentiation. J. Petrol. 51, 1891-1912 (2010).

21. Harney, D., Von Gruenewaldt, G. \& Merkle, R. The use of plagioclase composition as an indicator of magmatic processes in the Upper Zone of the Bushveld Complex. Mineral. Petrol. 56, 91-103 (1996).

22. Maila, R. P. Geochemistry of magnetitite layers in the Upper Zone of the Bushveld 
618

619

620

621

622

623

624

625

626

627

628

629

630

631

632

633

634

635

636

637

638

639

640

641

642

643

644

645

646

647

648

649

650

651

Complex, South Africa. Thesis, Univ. Witwatersrand (2015).

23. Vukmanovic, Z., Holness, M. B., Stock, M.J. \& Roberts, R. J. The creation and evolution of crystal mush in the Upper Zone of the Rustenburg Layered Suite, Bushveld Complex, South Africa, J. Petrol. 60, 1523-1542 (2019).

24. Sievwright, R. H., O’Neill, H. S. C., Tolley, J., Wilkinson, J. J. \& Berry, A. J., 2020, Diffusion and partition coefficients of minor and trace elements in magnetite as a function of oxygen fugacity at $1150^{\circ} \mathrm{C}$. Contrib. Mineral. Petrol. 175, 40 (2020).

25. Cawthorn, R. G. Cr and Sr: Keys to parental magmas and processes in the Bushveld Complex, South Africa. Lithos 95, 381-398 (2007).

26. Balan, E. et al. The oxidation state of vanadium in titanomagnetite from layered basic intrusions. Am. Mineral. 91, 953-956 (2006).

27. Fischer, L.A. The upper zone of the Bushveld Complex, South Africa: parental magma and crystallization processes. Thesis, Gottfried Wilhelm Leibniz University (2018).

28. Hayes, B., Ashwal, L. D., Webb, S. J. \& Bybee, G. M. Large-scale magmatic layering in the Main Zone of the Bushveld Complex and episodic downward magma infiltration. Contrib. Mineral. Petrol. 172, 13 (2017).

29. Sparks, R. S. J., Huppert, H. E., Kerr, R. C., McKenzie, D. P. \& Tait, S. R. Postcumulus processes in layered intrusions. Geol. Mag. 122, 555-568 (1985).

30. Tait, S. \& Jaupart, C. Compositional convection in a reactive crystalline mush and melt differentiation. J. Geophys. Res. 97, 6735-6756 (1992).

31. Manoochehri, S. \& Schmidt, M. W. Settling and compaction of chromite cumulates employing a centrifuging piston cylinder and application to layered mafic intrusions, Contrib. Mineral. Petrol. 168, 1091 (2014).

32. Tegner, C., Thy, P., Holness, M. B., Jakobsen, J. K. \& Lesher, C. E. Differentiation and compaction in the Skaergaard Intrusion. J. Petrol. 50, 813-840 (2009).

33. Yao, Z. S., Qin, K. Z., Wang, Q. \& Xue, S. Weak B-type olivine fabric induced by fast compaction of crystal mush in a crustal magma reservoir. J. Geophys. Res. Solid Earth 124, 3530-3556 (2019).

34. Sparks, R. S. J. \& Huppert, H. E. Density changes during the fractional crystallization of basaltic magmas: fluid dynamic implications. Contrib. Mineral. Petrol. 85, 300-309 (1984).

35. Nield, D. A. Onset of thermohaline convection in a porous medium. Water Resour. Res. 4, 553-560.

36. Butcher, A. R. \& Werkle, R. K. W. Postcumulus modification of magnetite grains in the 
652

653

654

655

656

657

658

659

660

661

662

663

664

665

666

667

668

669

670

671

672

673

674

675

676

677

678

679

680

681

682

683

684

685

upper zone of the Bushveld Complex, South Africa. Lithos 20, 247-260 (1987).

37. Finnigan, C. S., Breanan, J. M., Mungall, J. E. \& McDonough, W. F. Experiments and models bearing on the role of chromite as a collector of Platinum Group Minerals by local reduction. J. Petrol. 49, 1647-1665 (2008).

38. Crank, J. The mathematics of diffusion. Oxford University press, p. 414 (1979).

39. Costa, F., Dohmen, R. \& Chakraborty, S. Time scales of magmatic processes from modeling the zoning patterns of crystals. Rev. Mineral. Geochem. 69, 545-594 (2008).

40. Zhang, Y. X. Toward a quantitative model for the formation of gravitational magmatic sulfide deposits. Chem. Geol. 391, 56-73 (2015).

41. Cawthorn, R. G., McCarthy, T. S. \& Davies, G. Vertical chemical gradients in a single grain of magnetite from the Bushveld Complex, South Africa. Mineral. Mag. 47, $27-$ 34 (1983).

42. Grove, T. L., Baker, M. B. \& Kinzler, R. J. Coupled CaAl-NaSi diffusion in plagioclase feldspar: Experiments and applications to cooling rate speedometry. Geochim. Cosmochim. Acta 48, 2113-2121 (1984).

43. Nex, P. A. M., Cawthorn, R. G. \& Kinnaird, J. A. Geochemical effects of magma addition: compositional reversals and decoupling of trends in the Main Zone of the western Bushveld Complex. Mineral. Mag. 66, 833-856 (2002).

44. Cheadle, M. J., Elliott, M. T. \& McKenzie, D. Percolation threshold and permeability of crystallizing igneous rocks: The importance of textural equilibrium. Geology 32, 757760 .

45. Polacci, M. et al. Crystallisation in basaltic magmas revealed via in situ 4D synchrotron X-ray microtomography. Sci. Rep. 8,8377 (2018).

46. Wright, C. J., McCarthy, T. S. \& Cawthorn, R. G. Numerical modelling of trace element fractionation during diffusion controlled crystallization. Comput. Geosci. 9, 367-389 (1983).

47. Koepke, J. \& Behrens, H. Trace element diffusion in andesitic melts: an application of synchrotron X-ray fluorescence analysis. Geochim. Cosmochim. Acta 65, 1481-1498 (2021).

48. Maier, W. D., Barnes, S. J., Muir, D., Savard, D., Lahaye, Y. \& Smith, W. D. Formation of Bushveld anorthosite by reactive porous flow. Contrib. Mineral. Petrol. 176, 3 (2021).

49. Niu, Y. L. \& Batiza, R. In situ densities of MORB melts and residual mantle: Implications for buoyancy forces beneath Mid-Ocean Ridge. J. Geol. 99, 767-775.

50. Lesher, C. E. \& Spera, F. J. Thermodynamic and transport properties of silicate melts and 
686

687

688

689

690

691

692

693

694

695

696

697

698

699

700

701

702

703

704

705

706

707

708

709

710

711

712

713

714

715

716

717

magma. In: Sigurdsson, H. et al. (eds) The Encyclopedia of Volcanoes, $2^{\text {nd }}$. London Academic Press, 113-142 (2015).

51. Giordano, D., Russell, J. K. \& Dingwell, D. B. Viscosity of magmatic liquids: A model. Earth Planet. Sci. Lett. 271, 123-134.

52. McKenzie, 1985. The extraction of magma from the crust and mantle. Earth Planet. Sci. Lett. 74, 81-91.

53. Water, D. A. \& Watson, E. B. Grain-scale permeabilities of textually equilibrated, monomineralic rocks. Earth Planet. Sci. Lett. 164, 591-605.

54. Zhang, Y. X. \& Xu, Z. J. Kinetics of convection crystal dissolution and melting, with applications to methane hydrate dissolution and dissociation in seawater. Earth Planet. Sci. Lett. 213, 133-148.

55. Costa, A. 2006. Permeability-porosity relationship: A reexamination of the KozenyCarman equation based on a fractal pore-space geometry assumption. Geophys. Res. Lett. 33, L02318.

56. Gelhar, L. W., Welty, C. \& Rehfeldt, K.R., 1992, A critical review of data on field-scale dispersion in aquifers. Water Resour. Res. 28, 1955-1974 (1992).

57. DePaolo, D. J. High-frequency isotopic variations in the Mauna Kea tholeiitic basalt sequence: Melt zone dispersivity and chromatography. J. Geophys. Res. 101, 1185511864.

\section{Author contributions:}

J.E.M. and Z.-S.Y. discussed the concepts; Z.-S.Y. performed modeling calculations. J.E.M. and Z.-S.Y. wrote the manuscript.

\section{Competing interests:}

The authors declare no competing interests.

\section{Materials and correspondence:}

Requests should be addressed to J.E.M. 